\title{
THICK WINDS FROM WOLF-RAYET STARS
}

\author{
D. EICHLER \\ Department of Physics, Ben Gurion University, Beer Sheva, Israel \\ and
}

A. BAR SHALOM and Y. OREG

Nuclear Research Center, Beer Sheva, Israel

\begin{abstract}
When the effective opacity becomes sufficiently great, a qualitatively different type of radiatively driven wind solution appears, in which a high wind thickness and trapping of radiation cause each other in a bootstrap fashion. It is proposed that the thick winds from Wolf-Rayet stars can be understood in this manner. Opacity computations using the method of super transition arrays (STA) are presented that show that outflows from WR stars can attain the needed opacities.
\end{abstract}

We propose that the large mass outflow from Wolf-Rayet stars as compared with $O B$ stars is due to non-linearities in the hydrodynamics and opacitytemperature relation that allows small changes in parameters to alter the solution qualitatively. Slightly increasing the opacity just past a critical value, of order 10 times the Thomson scattering extinction, can cause a much higher mass loss. We derive opacities in the relevant range using the method of super transition arrays (STA). Due to the contribution of elements $\mathrm{Ne}$ through Fe, the Rosseland mean opacities can indeed be more than an order of magnitude higher than Thomson scattering for temperatures and densities that are likely to obtain at the critical radius of the outflow.

While the blackbody temperatures that would provide WR luminosities are only of the order of $60,000 \mathrm{~K}$, an excessive wind at the critical surface could cause photons to be trapped there. If the photons were completely trapped, and were to escape only via convection, the temperature at the critical surface $T_{c}$ would be of order $300,000 \mathrm{~K}$, and the opacity would at this temperature be too small to trap the photons. We therefore anticipate that the opacity is adjusted by the hydrodynamics; the material heats up until the opacity is reduced enough to alleviate the heating. It can be shown that a solution in which the optical depth of the wind, the resulting mass loss, temperature and opacity are computed self consistently requires that the effective opacity $\kappa_{e}$ (probably at least the Rosseland mean) obeys

$$
\kappa_{e} \sim(4 / 3 \alpha) \kappa_{T},
$$

where $\kappa_{T}$ is the Thomson scattering extinction per unit mass, and where $\alpha$ is the ratio of the luminosity to the Eddington luminosity $\left(L / L_{e d d}\right)$. If $\kappa_{e}>(4 / 3 \alpha) \kappa_{T}$, then, to within the accuracy of this theoretical treatment, the radiation is essentially trapped, and the photons are convected through 
the critical surface. The radiation force $F_{r}$ may then approach its maximum value $\nabla P_{r}$, and the mass loss rate can approach its maximum dynamically allowed value. If $\kappa_{e}<(4 / 3 \alpha) \kappa_{T}$, the photons freely stream from the critical surface, and allow a minimal $T_{c}$. When the opacity is too high to allow free streaming at minimal $T_{c}$, then we expect $T_{c}$ to adjust to establish the approximate equality $\kappa_{e} \sim(4 / 3 \alpha) \kappa_{T}$. We suggest that this is how WR winds work.

Typically $\alpha$ is observed to be about 0.1 to 0.4 for WR stars. We thus require $\kappa_{e} / \kappa_{T}$ to be of order 3 to 10 . For reasonable assumptions about the composition and temperature, the opacity can indeed be this high. We have computed opacities as a function of frequency, density and temperature for He-rich material with approximately cosmic abundances of $\mathrm{CNO}, \mathrm{Ne}, \mathrm{Mg}$, $\mathrm{Si}$, and Fe. A thirty percent admixture of $\mathrm{H}$ was assumed to test the role of $\mathrm{H}$ at low temperatures, but this was found to be unimportant. The calculation uses the STA method (Bar Shalom et al. 1989). This method groups different atomic configurations into "super-configurations", and computes the mean energy and the variance of the energy associated with a transition array between two super-configurations. The procedure converges to the correct value (i.e., to the value obtained when there is only one configuration per super-configuration) even when the number of configurations per super-configuration is large. Detailed comparison between the STA method and OPAL results (Iglesias \& Rogers 1990) shows the results to be virtually identical in all cases where such comparison has been made (Oreg, private communication).

The opacity is greatly enhanced by the large number of energy levels within a given configuration due to electrostatic interaction between electrons (as in de Silva et al. 1992). For $\Delta n=0$ transitions, the spread in energy among different levels due to electrostatic repulsion can be comparable to the energy of the transition. At $T_{c} \sim 100,000 \mathrm{~K}$, of the elements $\mathrm{Ne}, \mathrm{Mg}, \mathrm{Si}$ and $\mathrm{Fe}$ absorption is found to blanket the spectrum, providing an order of magnitude or more enhancement over the Thomson extinction at virtually all relevant frequencies. This is enough to trap the photons to the extent that $T_{c}$ and $\kappa_{e}$ are self-regulating as described above.

\section{References}

Bar Shalom, A. Oreg, J. Goldstein, W.H., Shvarts, D., Zigler, A. 1989, Phys. Rev. A 40, 3183

de Silva, L.B. et al. 1992, Phys. Rev. Lett. 69, 438

Igelsias, C.A., Rogers, F.J. 1990, in : W.H. Goldstein, C. Hooper, J. Ganthier, J. Seely, R. Lee (eds.), OPAL Opacities for Astrophysical Applications, Proc. 4th Int. Workshop on Radiative Properties of Hot Dense Matter (Singapore: World Scientific), p. 196 